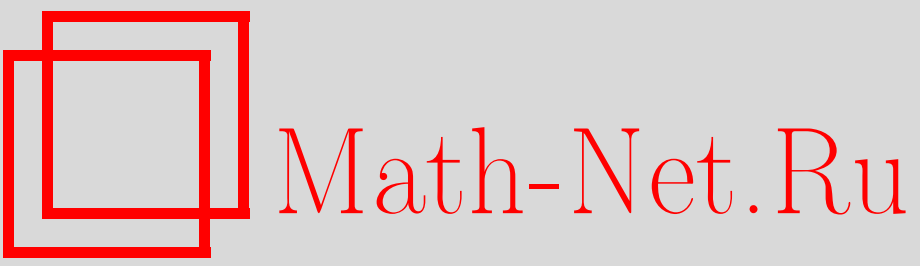

С. В. Козырев, Неархимедово пространство состояний в модели полимеризации, ТМФ, 1997, том 112, номер 3, 492-500

DOI: https://doi.org/10.4213/tmf1058

Использование Общероссийского математического портала Math-Net.Ru подразумевает, что вы прочитали и согласны с пользовательским соглашением

http://www . mathnet.ru/rus/agreement

Параметры загрузки:

IP : 54.162 .27 .143

26 апреля 2023 г., 16:59:18 


\author{
ТЕОРЕТИЧЕСКАЯ \\ И МАТЕМАТИЧЕСКАЯ \\ ФИЗИКА \\ Том 112, № 3 \\ сентябрь, 1997
}

С.В. Козырев*

\title{
НЕАРХИМЕДОВО ПРОСТРАНСТВО СОСТОЯНИЙ В МОДЕЛИ ПОЛИМЕРИЗАЦИИ
}

Рассматривается кинетика полимеризации, обусловленной процессами склейки и разрыва полимерных цепей. Показано, что на некотором пространстве возмущений над минимумом свободной энергии возникает топология 2-адического диска. Обсуждается применение к теории биологической эволюции.

\section{1. ВВЕДЕНИЕ}

Проблемы сложной химической кинетики являются предметом многочисленных обсуждений [1-5] в связи с проблемами биологической и предбиологической эволюции. В частности [4], высказывалось предположение, что в характерных для биофиизики системах должны реализовываться состояния с весьма специфическими свойствами.

В настоящей работе строится подобный набор состояний для системы реагируюших полимеров. Показано, что на рассматриваемом наборе состояний сушествует неархимедова метрика. Неархимедова метрика на пространстве возмушенных состояний имеет следуюший физический смысл. Каждое состояние модели характеризуется набором концентраций полимерных цепей. Вводимая метрика измеряет разность концентраций полимерных цепей в различных возмушенных состояниях.

Применение неархимедова анализа в математической физике являлось предметом книги [6].

Ультраметрические пространства возникали в математической физике в связи с изучением моделей спиновых стекол [7]. Ультраметрические пространства также возникают при рассмотрении свободных когерентных состояний [8], которые могут оказаться полезными при изучении моделей квантовой теории поля $[9,10]$.

Применение неархимедовых пространств к биологии обсуждалось в работах $[11,12]$. В частности, ультраметричность пространства биологических видов делает возможной таксономическую классификацию биологических видов $[11,12]$. Поскольку основные свойства биологических систем должны наследоваться с химического уровня описания, естественно искать обоснование ультраметричности пространства биологических видов, рассматривая кинетические уравнения, описываюшие химические реакции. В настоящей работе для модели обратимой полимеризации построен набор состояний, допускаюший неархимедову метрику.

\footnotetext{
* Институт химической физики РАН, Москва, Россия
} 
Одним из видов применения ультраметрических пространств в биологии может стать их приложение к проблемам биологической эволюции. В книге [3] строятся модели эволюции как случайного блуждания на ландшафте (пространстве видов). В работе [5] изучаются соответствующие кинетические уравнения, описывающие эволюцию как диффузию на пространстве видов. При этом применяется следующий подход. Если блуждаюшие частишы захватываются некоторой областью ландшаффта, то этой области ставится в соответствие биологический вид. Переходу частищ из одной такой области в другую соответствует возникновение нового вида. Блужданию ставятся в соответствие мутации. Ландшафрт соответствует “давлению отбора", т.е. одни области пространства видов являются более благоприятными, чем другие.

В таких моделях возникает проблема, называемая катастрофой ошибок [5]. Сушествует некоторое критическое значение скорости мутаций.

При скорости мутаций меньше критической блуждающие частицы захватьваются некоторыми областями ландшафта, но не могут при этом переходить в другие области. Это интерпретируется как возможность сушествования стабильных видов при низкой скорости мутаций. Стабильность вида в такой модели обеспечивается стабилизирующим отбором. При этом невозможно образование новых видов (т.е. эволюция).

При скорости мутаций больше критической частицы блуждают по всему ландшафту. Это интерпретируется как невозможность сушествования стабильных видов при высокой скорости мутаций.

Таким образом, в моделях, изученных в [5] и [3], без дополнительных предположений невозможны одновременное существование стабильных видов и образование новых видов.

Эта проблема может быть решена, если пространство видов считать ультраметрическим. Тогда стабильность вида могла бы быть обеспечена ультраметричностью пространства видов, т.к. в ультраметрическом пространстве невозможно выйти за пределы области, делая скачки длины меньше диаметра области $[12,13]$. При этом отпадает необходимость в стабилизирующем отборе, что соответствует концепции “эволюции без отбора" [14].

Рассматриваемая в настояшей работе модель основана на процессах склейки и разрыва линейных полимерных цепей. Рассматриваемая система описывается аналогом системы уравнений модели поликонденсации [15], кинетика которой описывается $F$-теоремой Айзенмана-Бека. $F$-теорема была доказана в работе [16] и состоит в следующем. Если в начальный момент времени концентрация полимерных последовательностей убывает достаточно быстро с ростом их длины, то система релаксирует к единственному устойчивому равновесному состоянию, являющемуся минимумом свободной энергии системы.

В качестве исследуемого набора состояний предлагается рассмотреть состояния, являющиеся возмущениями некоторого специального вида над минимумом свободной энергии системы.

Естественным образом определенная метрика, измеряюшая разность концентраций полимеров, на построенном пространстве возмущений будет неархимедовой.

\section{2. СИСТЕМА КИНЕТИЧЕСКИХ УРАВНЕНИЙ}

Модель Блатца-Тобольского поликонденсации линейных полимеров описывает ки- 
нетику полимеризации за счет процессов склейки и разрезания цепей. В этой модели имеется некий объем, содержащий раствор, в котором плавают линейные полимерные цепи. С этими цепями могут происходить следующие химические процессы. Процесс склейки состоит в склеивании концов цепей длин $i$ и $j$ в цепь длины $i+j$. Процесс разрыва состоит в разрьве цепи длины $k$ на цепи длин $i$ и $j, i+j=k$.

Пусть концентрация полимерной цепи длины $i, i=1,2 \ldots$ (количество цепей в единице объема), описывается неотрицательной функцией времени $n_{i}$. Тогда кинетические уравнения модели [15] имеют вид

$$
\frac{d}{d t} n_{k}=\sum_{i+j=k} n_{i} n_{j}-2 n_{k} \sum_{i=1}^{\infty} n_{i}+2 \sum_{i=1}^{\infty} n_{k+i}-(k-1) n_{k}
$$

Относительно решений этой системы см. [15-17], о связи системы (1) и уравнения Больцмана - $[16,17]$. В работе [16] для системы уравнений $(1)$ доказана $F$-теорема, являющаяся аналогом $H$-теоремы Больцмана.

Введем векторное пространство с базисом $\{[i]\}$, где базисный вектор $[i]$ соответствует последовательности длины $i$. Распределение концентраций различных последовательностей можно описать вектором концентрации

$$
\mathbf{n}=\sum_{i=1}^{\infty} n_{i}[i]
$$

здесь $n_{i}$ - концентрация последовательности $[i]$ длины $i$.

Используя вектор концентрации, систему уравнений (1) можно переписать в следующем виде:

$$
\frac{d}{d t} \mathbf{n}=-\sum_{i, j=1}^{\infty}\left(n_{i+j}-n_{i} n_{j}\right)([i+j]-[i]-[j])
$$

Для двух векторов вида (2), определенных формулами

$$
\begin{aligned}
& \mathbf{x}=\sum_{i=1}^{\infty} x_{i}[i] \\
& \mathbf{y}=\sum_{i=1}^{\infty} y_{i}[i]
\end{aligned}
$$

введем операцию свертки

$$
\mathbf{x} * \mathbf{y}=\sum_{i=1}^{\infty} x_{i} y_{i}[i]
$$

Функционал свободной энергии для рассматриваемой модели имеет вид

$$
F(\mathbf{n})=\sum_{i=1}^{\infty}\left(n_{i} \ln n_{i}-n_{i}\right)=e\left(\mathbf{n} * \sum_{i=0}^{\infty} \ln n_{i}[i]-\mathbf{n}\right),
$$

где линейный функционал $e$ определен формулой $e([i])=1 \forall i$. 
Одним из основных свойств модели является $F$-теорема, доказанная в работе [16]. $F$-теорема - аналог $H$-теоремы Больцмана, она утверждает, что функционал свободной энергии является функционалом Ляпунова, т.е. производная по времени значения этого функционала на решениях уравнения (3) неположительна и обрашается в нуль на единственном стационарном решении, а сам функционал ограничен снизу. Приведем формальное рассуждение, подтверждаюшее $F$-теорему.

УТВЕРЖДЕНИЕ 1. Если $\mathbf{n}=\sum_{i=1}^{\infty} n_{i}[i]$ удовлетворяет уравнению (3), то $\frac{d}{d t} F(\mathbf{n}) \leq 0$.

ДокАЗАТЕЛЬСТво. Нетрудно видеть, что

$$
\begin{aligned}
\frac{d}{d t} F(\mathbf{n}) & =\sum_{i=1}^{\infty} \frac{d}{d t} n_{i} \ln n_{i}=e\left(\frac{d}{d t} \mathbf{n} * \sum_{i=0}^{\infty} \ln n_{i}[i]\right)= \\
& =e\left(-\sum_{i, j=1}^{\infty}\left(n_{i+j}-n_{i} n_{j}\right)([i+j]-[i]-[j]) * \sum_{k=1}^{\infty} \ln n_{k}[k]\right)= \\
& =e\left(-\sum_{i, j=1}^{\infty}\left(n_{i+j}-n_{i} n_{j}\right)\left([i+j] \ln n_{i+j}-[i] \ln n_{i}-[j] \ln n_{j}\right)\right)= \\
& =-\sum_{i, j=1}^{\infty}\left(n_{i+j}-n_{i} n_{j}\right) \ln \left(\frac{n_{i+j}}{n_{i} n_{j}}\right) \leq 0 .
\end{aligned}
$$

Предметом настоящей работы является изучение упрощенной модели, соответствуюшей процессу обратимой полимеризации. Это означает, что приклеивание и отрезание происходят по одному мономеру. Уравнение такой модели имеет вид

$$
\frac{d}{d t} \mathbf{n}=-\sum_{i=1}^{\infty}\left(n_{i+1}-n_{i} n_{1}\right)([i+1]-[i]-[1]) .
$$

Для уравнения (7) и функционала (5) также имеет место утверждение 1.

Введем малый параметр $\epsilon>0$. Рассмотрим функционал $F_{\epsilon}$, являюшийся возмушением функционала свободной энергии $F$ вида

$$
F_{\epsilon}=\sum_{i=1}^{\infty}\left(n_{i} \ln (1+\epsilon) n_{i}-n_{i}\right) .
$$

Тогда $\lim _{\epsilon \rightarrow 0} F_{\epsilon}=F$,

$$
\frac{d}{d t} F_{\epsilon}(\mathbf{n})=-\sum_{i=1}^{\infty}\left(n_{i+1}-n_{i} n_{1}\right) \ln \left((1+\epsilon)^{-1} \frac{n_{i+1}}{n_{i} n_{1}}\right) .
$$

В качестве возмущенных состояний возьмем такой набор состояний системы $\mathbf{n}$, что $\frac{d}{d t} F_{\epsilon}(\mathbf{n})=0$. Мы будем называть подобные состояния стационарными состояниями функционала $F_{\epsilon}$. Эти состояния зависят от $\epsilon$ и при $\epsilon \rightarrow 0$ стремятся к минимуму свободной энергии. Основным утверждением работы является утверждение о существовании на заданном множестве состояний метрики, эквивалентной ультраметрической (две метрики эквивалентны, если порождают одинаковую топологию). 


\section{3. КОНСТРУКЦИЯ СТАЦИОНАРНЫХ СОСТОЯНИЙ}

В этом разделе будет построен некоторый набор стационарных состояний функционала $F_{\epsilon}$ для уравнения (7).

Состояние системы определяется набором концентраций $n_{i}, i=1,2, \ldots$. Стационарные состояния функционала $F_{\epsilon}$ задаются условием

$$
\sum_{k=1}^{\infty}\left(n_{k+1}-n_{k} n_{1}\right) \ln \left((1+\epsilon)^{-1} \frac{n_{k+1}}{n_{k} n_{1}}\right)=0
$$

Рассмотрим такие стационарные состояния функционала $F_{\epsilon}$, для которых каждый член этого ряда обращается в нуль. Получим систему рекуррентных уравнений

$$
\left(n_{k+1}-n_{k} n_{1}\right) \ln \left((1+\epsilon)^{-1} \frac{n_{k+1}}{n_{k} n_{1}}\right)=0 .
$$

Каждое уравнение этой системы имеет два решения:

$$
n_{k+1}^{(0)}=n_{1} n_{k}, \quad n_{k+1}^{(1)}=(1+\epsilon) n_{1} n_{k} .
$$

Введем строку индексов $U=u_{0} u_{1} \ldots$, параметризуюшую решения рекуррентного соотношения (9). Индексы, равные 0 и 1, расставляются следуюшим образом. Индекс $u_{k-1}$ равен 0 или 1 в зависимости от выбора для $n_{k+1}$ соответственно первого или второго решения уравнения (9).

В дальнейшем множество таких стационарных состояний функционала $F_{\epsilon}$ будем называть пространством возмущений. Состояние, отвечающее строке индексов $U$, будем обозначать $\mathbf{n}_{U}$.

Таким образом, в состоянии $\mathbf{n}_{U}$ имеем

$$
n_{k+2}=n_{1}^{k+2} \prod_{i=0}^{k}\left(1+\epsilon u_{i}\right)
$$

Для системы (7) имеет место закон сохранения количества вещества:

$$
\sum_{k=1}^{\infty} k n_{k}=M
$$

где суммарное число мономеров $M$ - константа. Решения рекуррентной системы (10) зависят от параметра $x_{U}=n_{1}$, который фиксируется законом сохранения (11).

Из (11) получаем, что $x_{U}$ есть корень уравнения

$$
x_{U}+\sum_{k=0}^{\infty}(k+2) x_{U}^{k+2} \prod_{i=0}^{k}\left(1+\epsilon u_{i}\right)=M .
$$


Нетрудно видеть, что для $\epsilon>0$ решение уравнения (12) принадлежит сегменту $\left[x_{0}, x_{1}\right]$, где

$$
\begin{aligned}
& x_{1}=1+\frac{1}{2 M}(1-\sqrt{1+4 M}) \\
& x_{0}=\frac{1}{1+\epsilon}\left(1+\frac{1}{2 M(1+\epsilon)}(1-\sqrt{1+4 M(1+\epsilon)})\right) .
\end{aligned}
$$

Введем функцию

$$
\eta_{U}(x)=x+\sum_{k=0}^{\infty}(k+2) x^{k+2} \prod_{i=0}^{k}\left(1+\epsilon u_{i}\right),
$$

описываюшую зависимость обшего количества мономеров в звеньях произвольной длины от количества свободных мономеров. Тогда закон сохранения (12) имеет вид $\eta_{U}(x)=M$.

Рассмотрим решения $x_{U}, x_{V}$ уравнения (12), отвечаюшие строкам индексов $U$ и $V$, соответственно.

Имеет место лемма.

ЛЕмма 1. Пусть строки $U$ u $V$ совпадают до индекса $k-1$ включительно, тогда

$$
\begin{aligned}
\left|x_{U}-x_{V}\right| \leq & \frac{\left(1-x_{0}\right)^{3}}{1+x_{0}} \frac{\epsilon x_{1}^{k+2}(1+\epsilon)^{k}}{\left(1-x_{1}\right)\left(1-x_{1}(1+\epsilon)\right)} \times \\
& \times\left(k+1+\frac{1-x_{1}^{2}(1+\epsilon)}{\left(1-x_{1}\right)\left(1-x_{1}(1+\epsilon)\right)}\right), \\
\left|x_{U}-x_{V}\right| \geq & \frac{\left(1-x_{1}\right)^{3}}{1+x_{1}} \frac{\epsilon x_{0}^{k+2}}{1-x_{0}}\left(k+1+\frac{1}{1-x_{0}}\right)
\end{aligned}
$$

ДокАЗАТЕЛЬСТво. Имеют место оценки

$$
\begin{aligned}
\left|\eta_{U}(x)-\eta_{V}(x)\right| \leq & \frac{\epsilon x^{k+2}(1+\epsilon)^{k}}{(1-x)(1-x(1+\epsilon))} \times \\
& \times\left(k+1+\frac{1-x^{2}(1+\epsilon)}{(1-x)(1-x(1+\epsilon))}\right) \\
\left|\eta_{U}(x)-\eta_{V}(x)\right| \geq & \frac{\epsilon x^{k+2}}{1-x}\left(k+1+\frac{1}{1-x}\right)
\end{aligned}
$$

Докажем оценку сверху для $\left|x_{U}-x_{V}\right|$. Из уравнения (12) имеем $\eta_{U}\left(x_{U}\right)=\eta_{V}\left(x_{V}\right)=M$, откуда

$$
\eta_{U}\left(x_{V}\right)-\eta_{U}\left(x_{U}\right)=\eta_{U}\left(x_{V}\right)-\eta_{V}\left(x_{V}\right),
$$

следовательно,

$$
\left|\eta_{U}\left(x_{V}\right)-\eta_{U}\left(x_{U}\right)\right|=\left|\eta_{U}\left(x_{V}\right)-\eta_{V}\left(x_{V}\right)\right|,
$$

при этом $\left|\eta_{U}\left(x_{V}\right)-\eta_{U}\left(x_{U}\right)\right| \geq \min _{x_{0} \leq x \leq x_{1}} \frac{d}{d x} \eta_{U}(x)\left|x_{U}-x_{V}\right|$.

Из оценки для $\left|\eta_{U}(x)-\eta_{V}(x)\right|$ получаем утверждение леммы. Аналогично доказывается оценка снизу. 


\section{4. ПОСТРОЕНИЕ НЕАРХИМЕДОВОЙ МЕТРИКИ}

В данном разделе будет доказана неархимедовость естественной метрики на введенном пространстве возмущений.

Рассмотрим следующую метрику на пространстве возмущений:

$$
\rho(U, V)=\lim _{\epsilon \rightarrow 0} \frac{1}{\epsilon} \sum_{i=1}^{\infty}\left|n_{i}(U)-n_{i}(V)\right|
$$

Эта метрика строится как главный член $l_{1}$-метрики при $\epsilon \rightarrow 0$. Предел при $\epsilon \rightarrow 0$ существует, т.к. соответствующий ряд мажорируется производной геометрической прогрессии.

Оценим эту метрику сверху и снизу.

Лемма 2. Пусть строки $U$ u $V$ совпадают до индекса $k-1$ включительно. Тогда существует набор констант $A, B, C, D>0$ таких, что

$$
\left(x_{0}\right)^{k}(A+k B) \leq \rho(U, V) \leq\left(x_{1}(1+\epsilon)\right)^{k}(C+k D) .
$$

ДокАЗАТЕЛЬСТво. Имеем следуюшую цепочку соотношений:

$$
\begin{aligned}
\sum_{i=1}^{\infty}\left|n_{i}(U)-n_{i}(V)\right|= & \left|x_{U}-x_{V}\right|+\sum_{k=0}^{\infty}\left|x_{U}^{k+2} \prod_{i=0}^{k}\left(1+\epsilon u_{i}\right)-x_{V}^{k+2} \prod_{i=0}^{k}\left(1+\epsilon v_{i}\right)\right| \leq \\
\leq & \left|x_{U}-x_{V}\right|+\sum_{k=0}^{\infty} x_{U}^{k+2}\left|\prod_{i=0}^{k}\left(1+\epsilon u_{i}\right)-\prod_{i=0}^{k}\left(1+\epsilon v_{i}\right)\right|+ \\
& +\sum_{k=0}^{\infty}\left|x_{U}^{k+2}-x_{V}^{k+2}\right| \prod_{i=0}^{k}\left(1+\epsilon v_{i}\right) \leq \\
\leq & \frac{\epsilon x_{1}^{k+2}(1+\epsilon)^{k}}{\left(1-x_{1}\right)\left(1-x_{1}(1+\epsilon)\right)}+\frac{\left|x_{U}-x_{V}\right|}{\left(1-x_{1}(1+\epsilon)\right)^{2}}
\end{aligned}
$$

Отсюда, применяя лемму 1 , получим оценку сверху для $\rho(u, v)$. Оценка снизу следует из леммы 1 и того, что $\rho(U, V)>\left|x_{U}-x_{V}\right|$.

В расмотренных выше леммах строки индексов $U$ и $V$ совпадают до $k-1$-го индекса включительно. Сопоставим этим строкам индексов 2 -адические числа $U=\sum_{i=0}^{\infty} u_{i} 2^{i}$, $V=\sum_{i=0}^{\infty} v_{i} 2^{i}$. Тогда расстояние между соответствуюшими 2-адическими числами равно $\|U-V\|_{Q_{2}}=2^{-k}$.

Теорема 1. Метрика $\rho(U, V)$ делает пространство возмущений топологическим пространством, гомеоморфным кольиу цельх 2-адических чисел. Соответствующий гомеоморфизм сопоставляет состоянию $\mathbf{n}_{U} 2$-адическое число $U=$ $\sum_{i=0}^{\infty} u_{i} 2^{i}$ 
ДокАЗАТЕльство. Метрика $\rho(U, V)$ в предыдуших леммах была оценена сверху и снизу метриками

$$
\rho_{1}(U, V)=\left(x_{0}\right)^{k}(A+k B), \quad \rho_{2}(U, V)=\left(x_{1}(1+\epsilon)\right)^{k}(C+k D),
$$

т.e.

$$
\rho_{1}(U, V) \leq \rho(U, V) \leq \rho_{2}(U, V)
$$

Метрики $\rho_{1}(U, V)$ и $\rho_{2}(U, V)$ являются ультраметриками. Это означает, что для этих метрик выполнено сильное неравенство треугольника

$$
\begin{aligned}
& \rho_{1}(U, V) \leq \max \left(\rho_{1}(U, W), \rho_{1}(V, W)\right) \\
& \rho_{2}(U, V) \leq \max \left(\rho_{2}(U, W), \rho_{2}(V, W)\right)
\end{aligned}
$$

для произвольных $U, V, W$. Метрики $\rho_{1}(U, V)$ и $\rho_{2}(U, V)$ имеют одинаковый набор открытых шаров. Следовательно, эти две метрики задают на пространстве возмушений одинаковую структуру топологического пространства.

Исследуем топологию на пространстве возмушений, задаваемую метрикой $\rho_{1}(U, V)$. Топология в метрическом пространстве определяется множеством всех замкнутых шаров. Множество замкнутых шаров $\left\{B_{U, k}\right\}$ в пространстве возмушений с метрикой $\rho_{1}(U, V)$ параметризуется парами $(U, k)$. Здесь $U-$ строка индексов, $k$ - неотрицательное целое число, шар $B_{U, k}$ содержит все состояния $V$ со строками $U$ и $V$, совпадаюшими до индекса $k-1$ включительно.

Отображение $\mathbf{n}_{U} \mapsto \sum_{i=0}^{\infty} u_{i} 2^{i}$ отображает шар $B_{U, k}$ на замкнутый шар в кольце целых 2-адических чисел радиуса $2^{-k}$ с центром в точке $\sum_{i=0}^{\infty} u_{i} 2^{i}$. Следовательно, отображение $\mathbf{n}_{U} \mapsto \sum_{i=0}^{\infty} u_{i} 2^{i}$ есть гомеоморфизм пространства возмущений с топологией, определенной метрикой $\rho_{1}(U, V)$, на кольцо целых 2 -адических чисел.

Из формулы (16) следует, что те же утверждения будут выполняться для топологии с метрикой $\rho(U, V)$.

Метрика свободного фоковского пространства делает пространство возмушений неархимедовым метрическим пространством, эквивалентным как топологическое пространство кольцу целых 2-адических чисел.

Автор благодарен В. А. Аветисову и И. В. Воловичу за обсуждения.

\section{Список литературы}

[1] V. A. Avetisov, V. I. Goldanskii. Proc. Nat. Acad. Sci. USA. 1996. V. 93. P. 11435-11442.

[2] Л. А. Блюменфельд. Проблемы биологической физики. М.: Наука, 1977.

[3] S. A. Kauffman. The origins of order. Oxford: Oxford University Press, 1993.

[4] А. Ю. Гросберг. Высокомолекулярные соединения. А. 1992. Т. 34. С. 174-180.

[5] M. Eigen, J. McCaskill, P. Shuster. J. Phys. Chem. 1988. V. 92. P. 6881-6891.

[6] В.С. Владимиров, И. В. Волович, Е. И. Зеленов. р-Адический анализ и математическая физика. М.: Наука, 1994.

[7] G. Parisi. Spin Glasses and Optimization Problem without Replicas, from Chance and Matter. In: Field Theory, Disorder and Simulations. Singapore: World Scientific Publishing, 1992.

[8] С. В. Козырев. ТМФ. 1997. Т. 110. С. 334.

[9] L. Accardi, Y. G. Lu. Wigner semicircle low in quantum electrodynamics. Preprint of Volterra Center № 126, 1992; Commun. Math. Phys. 1996. V. 168. P. 296. 
[10] I. Ya. Aref'eva, I. V. Volovich. The Master Field for QCD and $q$-deformed Quantum Field Theory. Preprint SMI-25-95; Nucl. Phys. B. 1996. V. 462. P. 600-612.

[11] G. Parisi. On the natural emergence of tree-like structures in complex systems. In: Perspectives on Biological Complexity. Eds O.T. Solbrig, G. Nicolis. IUBS, 1991; see also in: Field Theory, Disorder and Simulations. Singapore: World Scientific Publishing, 1992.

[12] R. Rammal, G. Toulouse, M. A. Virasoro. Rev. Mod. Phys. 1986. V. 58. P. 765-789.

[13] M. Mezard, G. Parisi, N. Sourlas, G. Toulouse, M. A. Virasoro. J. Phys. Lett. 1984. V. 45. P. 843.

[14] А. Лима-де-Фариа. Эволюция без отбора. Автоэволюция формы и функции. М.: Мир, 1991.

[15] P. L. Blatz, A. V. Tobolsky. J. Phys. Chem. 1945. V. 49. P. 77.

[16] M. Aizenman, T. A. Bak. Commun. Math. Phys. 1979. V. 65. P. 203.

[17] Неравновесные явления: уравнение Больцмана. Сб. статей. Ред. Дж. Л. Либовиц и Е. У. Монтролл. М.: Мир, 1986.

Поступила в редакцию 13.I.1997 г.

\section{S. V. Kozyrev \\ NONARCHIMEDEAN SPACE OF STATES IN THE MODEL OF POLYMERIZATION}

A system of reacting polymers with kinetics generated by reactions of recombination and degradation of polymer chains is considered. Space of excitations over the free energy minimum is constructed. Existence of a natural topology of 2-adic disc on this space of excitations is proved. Application to the theory of biological evolution is discussed. 